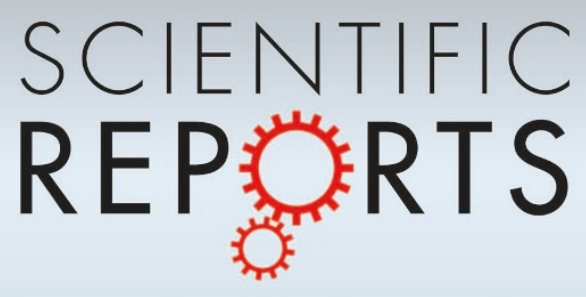

\title{
open Microwave Conductivity of Sorted CNT Assemblies
}

SUBJECT AREAS:

NANOSCALE MATERIALS

CARBON NANOTUBES AND

FULLERENES

Received

1 November 2013

Accepted

24 December 2013

Published

21 January 2014

Correspondence and requests for materials should be addressed to

K.K.K.K. (kk292@ cam.ac.uk)

\author{
John S. Bulmer' ${ }^{1}$ Jon Martens ${ }^{2}$, Lukasz Kurzepa' ${ }^{1}$ Tomasz Gizewski ' , M. Egilmez ${ }^{1,4}$, M. G. Blamire', \\ Noorhana Yahya ${ }^{3} \&$ Krzysztof K. K. Koziol'
}

'Department of Materials Science \& Metallurgy, University of Cambridge, 27 Charles Babbage Road, Cambridge CB3 OFS, UK,
${ }^{2}$ Anritsu Corporation, 490 Jarvis Dr Morgan Hill, CA 95037, ${ }^{3}$ Universiti Teknologi PETRONAS, Department of Fundamental and
Applied Science, Bandar Seri Iskandar, 31750, Tronoh, Perak, Malaysia, ${ }^{4}$ American University of Shariah, Department of Physics, UAE, PO26666.

Recent progress with tailored growth and post-process sorting enables carbon nanotube (CNT) assemblies with predominantly metallic or semi-conducting concentrations. Cryogenic and microwave measurements performed here show transport dimensionality and overall order increasing with increasing metallic concentration, even in atmospheric doping conditions. By $120 \mathrm{GHz}$, the conductivity of predominantly semi-conducting assemblies grew to $400 \%$ its DC value at an increasing growth rate, while other concentrations a growth rate that tapered off. A generalized Drude model fits to the different frequency dependent behaviors and yields useful quality control parameters such as plasma frequency, mean free path, and degree of localization. As one of the first demonstrations of waveguides fabricated from this material, sorted CNTs from both as-made and post-process sources were inserted into sections of practical micro-strip. With both sources, sorted CNT micro-strip increasingly outperformed the unsorted with increasing frequency-- illustrating that sorted CNT assemblies will be important for high frequency applications.

$\mathrm{n}$ the last twenty years, carbon nanotubes (CNTs) evolved from disordered soot on a transmission electron microscope grid $^{1}$, to pure, crystalline textiles capable of industrial scale production ${ }^{2,3}$. Contemporary chemical vapor deposition reactors (CVD) generate ensembles of aligned CNTs into bulk assemblies in a single processing step. Latest CVD advancements yield assemblies with single wall CNT (SWCNT) signatures ${ }^{4,5}$ and, under the right growth conditions, the preferential growth of metallic chiralities has been demonstrated ${ }^{6}$. In general, floating catalyst CVD produces CNTs that individually span on order of a millimeter ${ }^{2}$. This is far longer than other competing growth methods and is an advantage in electrical transport ${ }^{7}$ when one considers that most of the room temperature resistivity ( $\sim 95 \%)$ originates not from the CNTs, but the junctions between them ${ }^{8-12}$. In the best scenario, these CNT junctions raise thermally activated barriers to delocalized charge carriers. In the worst, these junctions localize the charge carriers attempting to ride the CNT railways ${ }^{11,13}$.

Alongside the development of one-step CNT fibre production, post-process sorting between SWCNT electronic structure has also evolved ${ }^{14-16}$. Where researchers grudgingly accepted polydisperse assemblies of typically one third metallic to two thirds semi-conducting, now post-process methods sort to any specified electronic concentration in research scale quantities. The transport mechanisms of such sorted assemblies are highly dependent on metallic concentration. A completely semi-conducting SWCNT assembly has electrical transport governed by one dimensional variable range hopping (1D VRH) where charge carriers localize and long range Coulomb interactions broaden the gap in the density of states. Increasing the assembly's metallic concentration increases the hopping dimensionality- a 35\% metal concentration has 2D VRH transport and a $74 \%$ metal concentration has 3D VRH transport. A fully metallic SWCNT assembly on the other hand supports truly delocalized transport where the VRH model could not be applied. Its room temperature conductivity approached a thousand times that of the fully semi-conducting assembly and illustrates that the semi-conducting SWCNTs are not just innocuous dead weight- their inclusion increased the overall material disorder, localized the charge carriers, and encumbered the transport ${ }^{11}$.

AC conductivity is a metric particularly sensitive to disorder and as we will show can probe the intrinsic transport parameters of a CNT assembly. In the microwave wavelengths, several groups ${ }^{12,17,18}$ showed that typical, unsorted SWCNT assemblies has a conductivity that increases with frequency according to a power law, which is typical of disordered materials in genera $1^{19,20}$. The disorder from these unsorted SWCNT films was attributed to CNT junctions or topological defects. This universal disorder model has not been completely successful however 
for all CNT groups exploring the microwave regime ${ }^{12,21,22}$ or, as we will see, for our measurements with sorted SWCNT assemblies. As microwave wavelengths shorten to $\mathrm{THz}^{23-28}$ and far infrared ${ }^{29-33}$ wavelengths, a more complicated AC response is observed with an AC conductivity that first grows with frequency to a peak (somewhere between $0.5-10 \mathrm{THz}$ ) and then decays away. Researchers often explain this with some combination of effective medium theory (EMT), traditional Drude conduction, Lortentz oscillators, and Plasmon resonances. As shown by at least one group ${ }^{28}$ however, $\mathrm{THz}$ conductivity is modeled by a generalized Drude mechanism (the Drude-Smith model) that adds only one extra parameter to typical Drude conduction that accounts for the degree of charge carrier backscatter.

In this communication we compare the microwave transmission of practical micro-strip waveguides with SWCNT assemblies inserted into the central conductor. The SWCNTs are sorted for metallic SWCNT concentration and originate from either floating catalyst CVD or post process sorting. Although this technique is limited to about $10 \mathrm{GHz}$, it immediately shows the advantage in transmission of sorted SWCNT assemblies over unsorted, without additional processing or complications dealing with film thickness. Next we use a standard contactless technique to calculate the bulk microwave conductivity of these assemblies up to $120 \mathrm{GHz}$. The response of predominantly semi-conducting SWNCTs is distinct to the other concentrations and does not follow power law behavior. We consider several AC conduction models and find that generalized Drude conduction, now applied in the microwave regime, fits for all the various metallic SWCNT concentrations.

Like AC transport, DC cryogenic transport is also especially sensitive to disorder as various mechanisms of conduction freeze out. At the lowest temperatures, only hopping mechanisms remain which is a direct probe into the material's order and transport dimensionality. We will explore the sorted material's DC response at cryogenic temperatures and observe the relationship to room temperature, microwave transport.

\section{Results}

The unaligned, but highly purified SWCNT films or "buckypaper" from NanoIntegris Inc. (called NI films (Fig. 1a)) were post process sorted for the following metallic concentration: $98 \%$ metallic, $98 \%$ semi-conducting, and the typical unsorted ratio. For the aligned floating catalyst CVD material developed here at Cambridge University (called UCAM material (Fig. 1b)), there were two types: $70 \mu \mathrm{m}$ wide fibre consisting of predominantly metallic SWCNTs ${ }^{6}$ and films with SWCNT signatures, but consisted of the typical unsorted electronic concentration.

Micro-strip transmission. As a practical test for microwave conductivity, each CNT assembly was integrated into a section of micro-strip transmission line (Fig. 1c). This technique immediately shows the transmission advantages of sorted SWCNT assemblies without additional processing or complications dealing with film thickness required by traditional microwave conductivity methodologies. Fig. 2a shows insertion loss $\left(I L=20 \log S_{21}\right)$ of the various NI films. $I L=0$ implies $100 \%$ power transmission through the micro-strip. The red dots indicate the expected insertion loss from a computer based micro-strip simulation. As illustrated, there is a difference with the transmission between sorted and unsorted films. The unsorted NI films match the predicted behavior of the model-monotonically decreasing insertion loss, as what would be expected from a lumped element resistor. For the sorted NI films, around $3 \mathrm{GHz}$ the measured insertion loss deviates from the model and levels off with increasing frequency.

Next we inserted UCAM material into the micro-strip. The unsorted UCAM material follows the typical, monatomic behavior of the transmission line model. The predominantly metallic UCAM material, however, has an insertion loss that first improves and then levels off with frequency (Fig. 2b). Note that this predominantly metallic UCAM material has both more efficient insertion and return losses compared to the unsorted UCAM material. This is despite the fact its $70 \mu \mathrm{m}$ fibre diameter does not match the exact geometry of the $0.5 \mathrm{~mm}$ wide transmission line. The unsorted UCAM material matches the geometry of the transmission line and follows the transmission line model. Its transmission however is more lossy in both insertion and return losses. The relatively low return losses (Fig. 2c) of the predominantly metallic UCAM material indicates most incident power is not reflected back, and shows the model deviation is likely an intrinsic material response rather than an impedance mismatch manifested by geometry irregularity. Insertion loss improving with frequency has been observed by other researchers, notably with

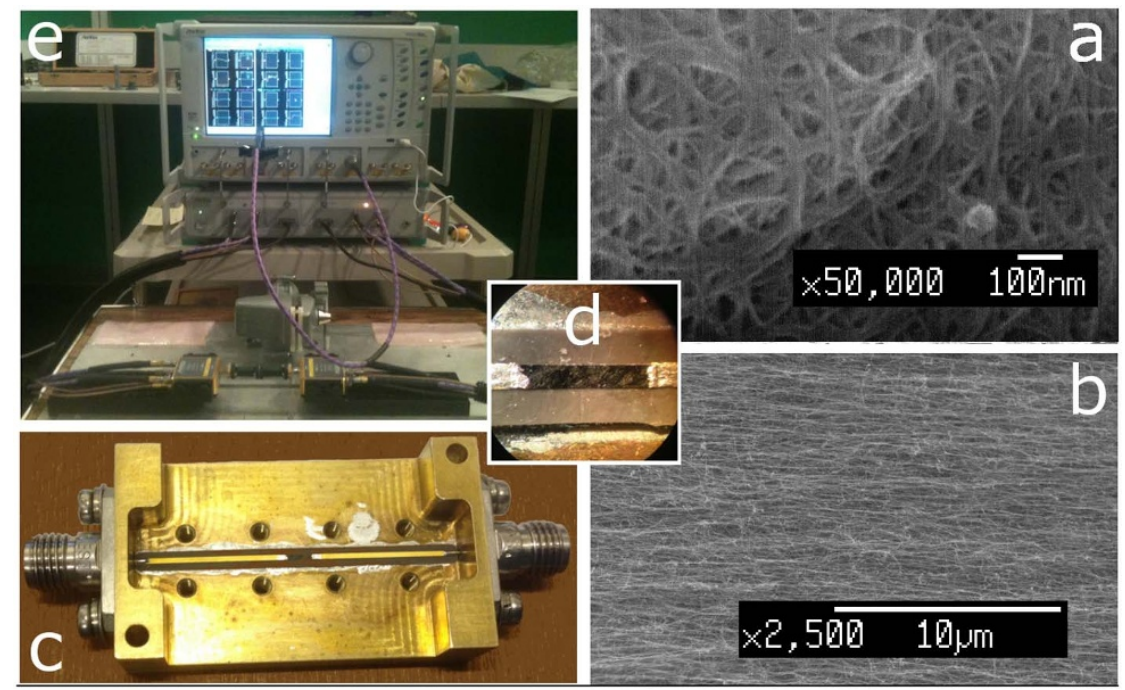

Figure $1 \mid$ (a) SEM image showing the unaligned nature of the NI film. The bundles are $\sim 12 \mathrm{~nm}$. (b) SEM image of the UCAM material showing vertical alignment and lower density compared to the NI film. (c) and (d) Sorted SWCNT material was inserted into a section of $50 \Omega$ micro-strip. A fitted shield is bolted onto the top to reduce radiation losses. (e) Setup showing the Nicolson, Ross, Weir (NRW) procedure with the SWCNT film sandwiched between rectangular waveguides, perpendicular to the transmission. An Anritsu VectorStar Vector Network Analyzer (VNA) with millimeter wave modules collected the $\mathrm{S}$ parameters. These $\mathrm{S}$ parameters were converted into a frequency dependent conductivity. 

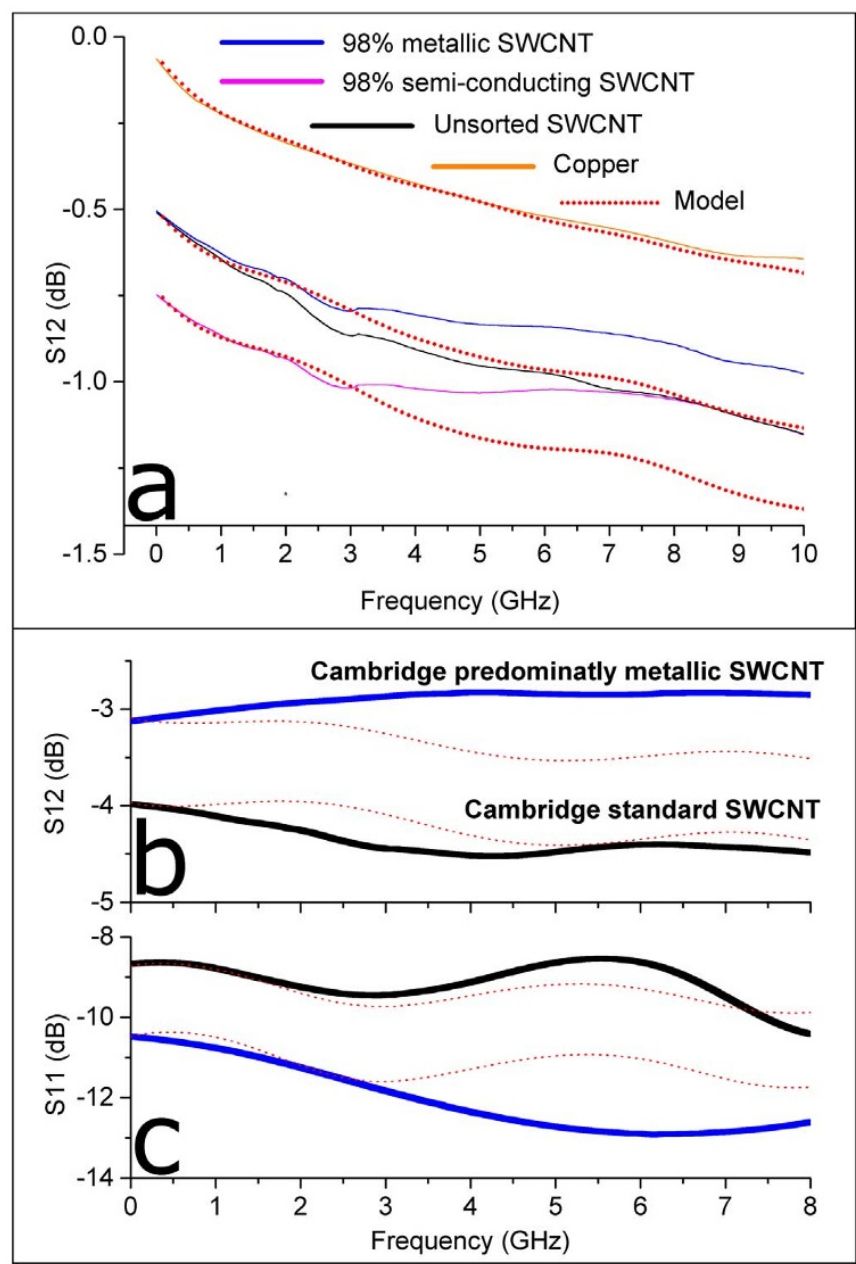

Figure $2 \mid$ (a) Expected (in red dots) and measured insertion loss of the NI films with various concentrations of metallic and semi-conducting SWCNTs. Insertion loss (b) and return loss (c) of the predominantly metallic and standard UCAM material, with red dots depicting the expected behavior.

co-planar transmission lines constructed from SWCNTs embedded in polymers ${ }^{34}$.

A frequency dependent sheet conductance was extracted from the insertion loss. Our approach does this without taking into account film thickness, which is often an inconsistent parameter in CNT assemblies and one that strongly impacts other microwave conductivity measurements. The sorted material in both the NI film and UCAM material has a sheet conductance that increases with frequency (Fig. 3). The low frequency results agrees with two probe DC measurements obtained with a Keithley multimeter (shown with crossed squares), with the greatest disagreement ( $20 \%)$ being with the metallic materials.

Application-wise this is one of the first demonstrations of microstrip waveguide manufactured with sorted SWCNT material. The advantage of sorted SWCNT material is more efficient microwave transmission that improves with increasing frequency. This is opposite the behavior of traditional metals that suffer a conductivity decrease arising from skin effect. The fact that the predominantly metallic UCAM material also has increasing microwave conductivity over the unsorted UCAM material suggests that this behavior applies to a wide range of sorted bulk SWCNT assemblies and not just the sorted NI films.

A challenge of this micro-strip technique is that the transmitting field is primarily present in the dielectric and the material under test

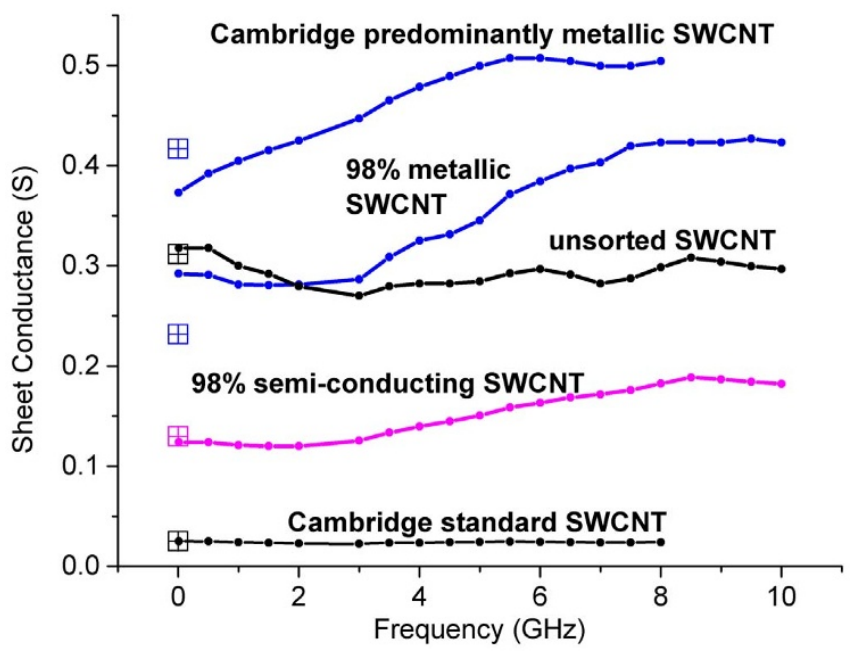

Figure 3 Extracted sheet conductance of the sorted NI films and UCAM material. Crossed boxes are DC values as measured by a Keithly multimeter. For both the NI films and UCAM material, the sorted variety has a conductivity that improves with frequency. Note that the unsorted SWCNTs has a sheet conductance comparable to the metallic SWCNTs only because the unsorted film is approximately eight times thicker.

is only at the boundary of this field. Other factors, such as the dielectric, affect the overall micro-strip performance and possibly complicate the extraction of a frequency dependent conductivity. Also, as conductivity increases with frequency, film inductance becomes increasingly significant. A $1 \mathrm{~mm}$ section of SWCNT micro-strip has an inductance of $\sim 0.2 \mathrm{nH}$, which at $10 \mathrm{GHz}$ has a reactive impedance contribution of $\sim 12 \Omega$ and increasing. By this point, the extrapolated resistance is $\sim 4 \Omega$ and possibly still falling. It is not clear if the level off in conductivity approaching $10 \mathrm{GHz}$ is due to the AC effect diminishing or sensitivity loss in the micro-strip. In order to determine the exact cause of the conductivity increase, we focus on the NI films with a standardized electrode-less microwave technique that probes greater bandwidths and does not have this inductance limitation.

Microwave conductivity to $120 \mathrm{GHz}$. We inserted the NI films perpendicular to the path of various waveguides and measured the conductivity from 16 to $120 \mathrm{GHz}$. In this range both the sorted and unsorted conductivity increases with frequency (Fig. 4). The 98\% metallic NI film increased $200 \%$ over this range and the unsorted NI film, 300\%. The conductivity growth in both materials tapered off at the high end of the spectrum. In the previous micro-strip measurements, the conductivity increase of the unsorted NI film was not apparent and this is likely because of a delay in the onset frequency with the universal disorder model discussed later. The conductivity of the $98 \%$ semi-conducting NI film grew the most at $400 \%$. Its conductivity growth distinctly increased at an increasing rate. For all three NI films, the conductivity at the low end of the spectrum was roughly congruent with the four probe DC conductivity measured from the Keithley multimeter.

\section{Discussion}

It has been shown that the AC conductivity of unsorted SWCNT films increases with frequency ${ }^{12,17,18,21}$ according to the extended pair power law ${ }^{19,20} \sigma(\omega) / \sigma_{0}=1+k\left(\omega / \omega_{0}\right)^{s}$ where $k$ is a fitted parameter, $\omega$ is frequency, $\omega_{0}$ is the onset frequency, and $s$ is a parameter $(0<s<1)$ that is related to the material's disorder. The greater the value of $s$, the greater the level of disorder with unity being the maximum limiting value. This universal disorder model is attractive because it does not specify how exactly the disorder is present, only that it exists. For CNTs, this disorder can be not just the polydisperse collections of 


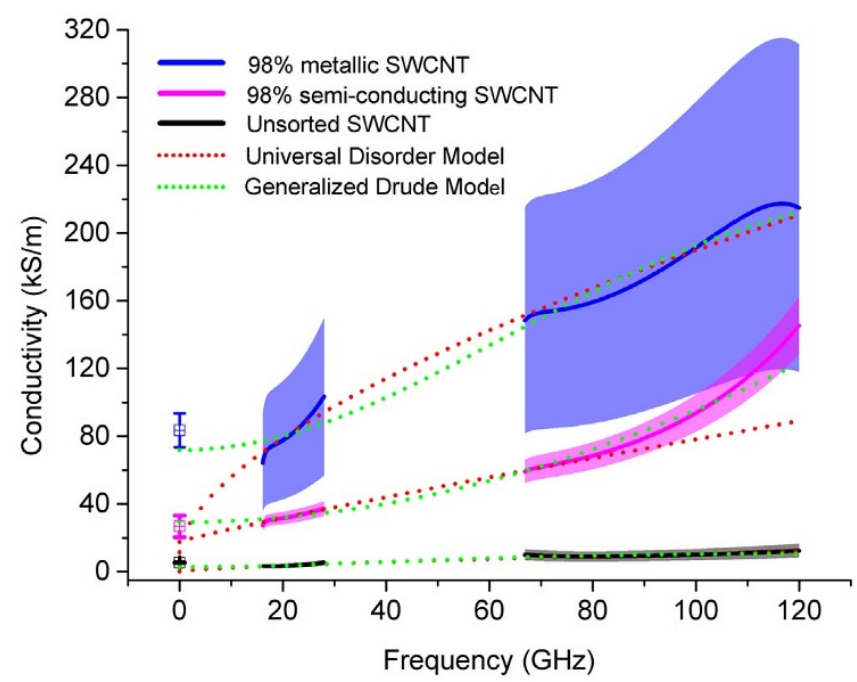

Figure $4 \mid$ Microwave conductivity using type $\mathrm{K}$ and $\mathrm{W}$ waveguides. The red dots are the fitted universal disorder model, which does not fit well with the $90 \%$ semi-conducting SWCNT film. The generalized Drude model is shown with green dots, which fits better to the three different film concentrations and agrees with the DC conductivity from a four probe multimeter. The estimated error, based on the film thickness variance, is displayed in the various colored bands.

various CNTs, but also disorder from junctions, defects and lack of internal alignment. With the NI films, the CNTs are unaligned and differences in disorder amongst the different NI films are presumably from different metallic to semi-conducting ratios. Note however that the average CNT length of the metallic NI film is half that of the semiconducting and unsorted NI films.

The universal disorder model yielded realistic fits to both the $98 \%$ metallic NI and unsorted NI microwave conductivities (Fig. 4 and Table 1a). Here, under the universal disorder model, the AC conductivity is proportional to its DC conductivity and explains why the 98\% metallic NI sample has the greatest absolute conductivity increase. The $98 \%$ metallic NI film has a smaller disorder exponent $(s=0.60)$ then the unsorted NI film $(s=0.67)$, which is expected due to greater order from the sorted electric structure. The difference of $s$ between the two is not great however and implies that the overall source of disorder is not just different metallic concentrations, but other sources such as junctions, defects and lack of internal alignment. For comparison, Table 1 also includes past work where the universal disorder model has been applied to unsorted and unaligned CNT materials. The highest conductivity SWCNT films have a somewhat lower $s$ value then our NI films and have an average SWCNT length four to eight times greater than the NI average ${ }^{18}$. Another group reported an $s$ value for their unsorted SWCNTs equal to our unsorted NI film $s$ value $^{17}$ and their average CNT length concurs with our unsorted NI CNT length.

The $98 \%$ semi-conducting NI film tells a different story. The best fit to the universal disorder model only applies up to $68 \mathrm{GHz}$ at the leading edge of the $\mathrm{W}$ band. Up to this point, the $s$ value obtained is $s$ $=0.91$ and, because $0.8 \leq s \leq 1.0$, this indicates a hopping conduction mechanism ${ }^{21}$. Note this $s$ value is greater than for the unsorted NI film, despite the fact that the unsorted NI film has a greater variety of electronic constituents. Beyond $68 \mathrm{GHz}$, the conductivity increases at an increasing rate and the universal disorder model cannot apply. A similar situation have been shown where the AC conductivity of unsorted SWCNT films follows the universal disorder model to a point, but then also begins to grow at an increasing rate. It's concluded a more complicated model is required, but the reports stop short at providing one $\mathrm{e}^{12,21}$.

Looking at other AC conduction mechanisms beyond the microwave regime, particularly explored with time domain $\mathrm{THz}$ spectroscopy $^{23-28}$ and far-infrared spectroscopy ${ }^{29-33}$, researchers have shown that AC conductivity typically increases to a point and then decreases at a slower rate. This exact location of this $\mathrm{THz}$ conductivity peak varies from sample to sample, but ranges from approximately $500 \mathrm{GHz}$ to $10 \mathrm{THz}$. Some studies ${ }^{23-27,29}$ describe this behavior with an effective medium theory (EMT) that calculates an effective permittivity based on a weighted average of the permittivity of the CNT network $\left(\varepsilon_{C N T}\right)$ and the air that surrounds it $\left(\varepsilon_{0}\right) . \varepsilon_{C N T}$ itself is typically calculated with two contributions: one from the traditional Drude model and the other from a Lorentz oscillator, which is with some controversy attributed to a CNT curvature induced band gap $(\sim 20 \mathrm{meV})$ present in non-armchair metallic CNTs. Recent work with CNTs films of different diameters support that indeed CNT curvature affects the $\mathrm{THz}$ conductivity peak ${ }^{30}$. Our microwave conductivity was probed with a maximum photon energy of $h f=$ $0.5 \mathrm{meV}$ and would not be energetic enough to overcome this band gap and lead to the $400 \%$ increase in conductivity at $120 \mathrm{GHz}$.

The curvature induced band gap explanation is controversial with other researchers ${ }^{31-33}$ demonstrating that the $\mathrm{THz}$ conductivity peak stems from resonances associated with Plasmon hybridization-a concept which has now been applied for a wide variety of nanogeometries ${ }^{35-37}$. This explanation is supported by researchers finding the position of the $\mathrm{THz}$ conductivity peak shifting to higher

\begin{tabular}{|c|c|c|c|}
\hline & Disorder Exponent $s$ & CNT length $(\mu \mathrm{m})$ & DC Cond. $(\mathrm{kS} / \mathrm{m})$ \\
\hline Arc discharge Unaligned SWCNT ${ }^{18}$ & 0.57 & $2-4$ & 50 \\
\hline HiPco SWCNT ${ }^{17}$ & 0.67 & $0.1-1.0$ & Contactless, N/A \\
\hline Unsorted NI (R2: 0.98) & $0.67+/-0.04$ & $\begin{array}{c}0.1-4 \\
\text { Average: } 1.0\end{array}$ & 5.4 \\
\hline $98 \%$ semi-conducting $\mathrm{NI}\left(\mathrm{R}^{2}: 0.99\right)$ & $0.91+/-0.03$ & $\begin{array}{c}0.1-4 \\
\text { Average: } 1.0\end{array}$ & 26.8 \\
\hline
\end{tabular}


frequency as average CNT length is reduced ${ }^{32}$. Very recently, an absorbance spectroscopy study ${ }^{33}$ ranging from the THz to the ultraviolet regime was conducted on CNT films sorted for metallic and semi-conducting content. It was found that the $\mathrm{THz}$ peak was present for both the metallic and (contradicting the curvature induced band gap) semi-conducting CNT films. Only after annealing the semi-conducting CNT film was the $\mathrm{THz}$ peak repressed, which again supports the Plasmon explanation. They model their AC conductivity with a traditional Drude contribution combined with a Plasmon resonance term. Using this model and their fitted parameters, however, a significant conductivity increase is only present in the $\mathrm{THz}$ regime and conductivity remains relatively constant in the microwave regime where our measurements were made.

This $\mathrm{THz}$ conductivity peak, either stemming from the curvature induced band gap or Plasmon resonance, does not explain the microwave conductivity increase (beyond the universal disorder model) observed by us measuring sorted CNT materials or others measuring unsorted CNT materials ${ }^{12,21}$. As been previously noted by others ${ }^{12}$, there are multiple conduction mechanisms that lead to distinct AC conductivity increases in both the microwave regime and $\mathrm{THz}$ regime. Between these two conductivity increases, it was reported ${ }^{12}$ that conductivity decreases due to the traditional Drude conduction. Traditional Drude behavior has been observed in the millimeter ${ }^{22}$ and $\mathrm{THz}$ regimes ${ }^{25}$ in a few isolated cases and, interestingly, has been with relatively high conductivity CNT films. To recap, our microwave conductivity of sorted CNT material is not explained with the power law behavior of the universal disorder model (like others) or the conductivity peak in the $\mathrm{THz}$ regime.

Another model-the generalized Drude model by $S m_{i t h}{ }^{38}$-has also been applied in the $\mathrm{THz}$ regime for sorted SWCNTs ${ }^{28}$.

$$
\sigma(\omega)=\frac{\varepsilon_{0} \omega_{p}^{2} \tau_{s}}{\left(1-i \omega \tau_{s}\right)}\left(1+\frac{c}{\left(1-i \omega \tau_{s}\right)}\right)
$$

with $\varepsilon_{0^{--}}$the vacuum permittivity, $\omega_{p^{--}}$the plasma frequency, $\tau_{s^{-}}$the average carrier scattering time between charge carrier collisions, and $c$ is the "persistence of velocity" or backscatter parameter with $-1 \leq$ $c \leq 0$. When $c=0$, this becomes the traditional Drude model and no charge carriers are backscattered. When $c=-1$, this means all charge carriers are localized and completely backscattered. Outside of CNTs, generalized Drude conduction has been applied in the $\mathrm{THz}$ and infrared regimes to other materials such as $\mathrm{Hg}$ and Titanium Dioxide $^{38,39}$. Although the model appears to have yet been applied to CNTs in the microwave regime, generalized Drude conduction is attractive because it is simpler then the EMT approach (five additional parameters beyond the Drude model) and Plasmon resonance approach (four additional terms beyond the Drude model), but has the freedom to describe the observed conduction growth (and possible eventual decrease) that the universal disorder model cannot allow. Also, this model, unlike the others, accounts for the contribution of localized charges that do not add to the DC conductivity, but may add to the AC conductivity at sufficiently high frequency. On a practically basis, finding the values of $\omega_{p}, \tau_{s}$, and $c$ is important to ascertain fundamental CNT conduction characteristics.

Displayed in green dots is the generalized Drude model fitting to all three conductivities, to include the increasing growth rate of the 98\% semi-conducting film (Fig. 4). Extrapolating the low frequency traces to their DC value, there is agreement with the model and what was measured with the four probe multimeter. In contrast, as depicted in red dots, the universal disorder model shot well below their DC values. Displayed in table 2 are the fitted parameters from the generalize Drude model. Using a Fermi velocity ${ }^{28}$ of $v_{f}=$ $8^{*} 10^{5} \mathrm{~m} / \mathrm{s}$, the mean free path distance is $0.7,1.0,0.2 \mu \mathrm{m}$ for the 98\% metallic (Average SWCNT length $=0.5 \mu \mathrm{m}$ ), unsorted (Average SWCNT length $=1 \mu \mathrm{m}$ ), and $98 \%$ semi-conducting (Average SWCNT length $=1 \mu \mathrm{m}$ ) NI films respectively. For the 98\% metallic and unsorted NI films, these mean free paths agree with their SWCNT lengths and imply that junctions are the primary source of backscattering. This is not the case for the $98 \%$ semi-conducting NI film where the mean free path is five times smaller than its average CNT length. Further, the backscatter parameter $c$ is much closer to -1 then the unsorted and metallic material and implies greater localization.

For comparison table 2 shows previous work ${ }^{28}$ on sorted SWCNT film conductivity fitted to the generalized Drude model as measured in the $\mathrm{THz}$ regime with time domain $\mathrm{THz}$ spectroscopy. These are unaligned SWCNT films of either $93 \%$ metallic or $94 \%$ semi-conducting electronic concentration and, as in our case, are in the "as-is" condition with unintentional doping from atmospheric exposure. While their plasma frequency and backscatter parameter $c$ is in the same order of magnitude as our VNA measured parameters, their mean free path was significantly less than our values. Possibly, this smaller scattering time is from shorter CNTs or defects causing multiple scattering sites on individual SWCNTs. Also included in table 2 is a millimeter wave study on unsorted and unaligned SWCNT film ${ }^{22}$. Their conductivity data fit to the traditional Drude model, which implies $c=0$.

Like AC transport, cryogenic transport is particularly sensitive to disorder as other conduction mechanisms freeze out and only variable range hopping $(\mathrm{VRH})$ mechanisms remain. Like the universal disorder model for $\mathrm{AC}$ conduction, this exact nature of this disorder is not necessarily specified in $\mathrm{VRH}$, but in our case is assumed to be from lack of internal CNT alignment, the contribution of CNT junctions and other defects, and different electronic SWCNT species present in the material. We implemented cryogenic, DC measurements to probe the material's disorder and shed light on the room temperature, microwave behavior. As discussed in the introduction, a previous study ${ }^{11}$ showed that increasing the metallic SWCNT concentration increases the dimensionality of transport in the VRH model. They included an atmospheric purging that vacuum annealed the SWCNT film at $500^{\circ} \mathrm{C}, 10^{-6}$ Torr, for 1 hour; trapped atmospheric constituents, such as water and oxygen, are known to modify CNT electronic structure ${ }^{40-42}$. Our microwave conductivity measurements were measured as-is without this atmospheric purging step and it was a question if the previous study's results could be applied in our case. We found, however, that even without this atmospheric purging, our sorted NI films' VRH dimensionality matched the previous study's results (Fig. 5). That is, the $98 \%$ semi-conducting NI film fitted ES-VRH, the unsorted NI film fitted to 2D VRH, and the 98\% metallic film did not follow any VRH model, but coincided with quantum transport limited by $2 \mathrm{D}$ weak localization with a resistance depending linearly on the natural log of temperature, $\ln (T)^{11,43,44}$. The other film concentrations distinctly did not fit to this relationship. The primary difference between the previous study and our results here was a significantly lower characteristic temperature in the VRH models, $T_{\text {Mott }}$, which is indicative of substantial doping and presumably this originates from atmospheric exposure ${ }^{43}$.

Qualitatively, the microwave measurements agree with the VRH dimensionality. All of the samples exhibited a negative $d R / d T$ indicative of hopping type of transport mechanism. The ES-VRH conduction in the $98 \%$ semi-conducting NI film implies $1 \mathrm{D}$ hopping down the SWCNT. Here, any degree of disorder produces localization because electrons lack the freedom to bypass a particularly insulating regime for another. The coulomb interaction between the localized electrons leads to a gap in the density of states in the conduction band ${ }^{11,44}$. Notice that distinctly high disorder parameter $s$ deduced from the universal disorder model also revealed a possible electron hopping mechanism.

The generalized Drude model on the $98 \%$ semi-conducting NI film showed a distinctly greater degree of electron backscatter as well as a distinctly smaller mean free path $(180 \mathrm{~nm})$ well within the length of the semi-conducting SWCNT. Thus, the material's disorder impacts the DC transport at cryogenic temperatures as 
Table 2 | In red, fitted generalized Drude conduction parameters for the various NI films. In black, past fits of this model to related materials. This table is sorted by DC conductivity

\begin{tabular}{|c|c|c|c|c|}
\hline & $\omega_{p} / 2 \pi(\mathrm{THz})$ & $\mathrm{mfp}(\mu \mathrm{m})$ & c & $D C$ cond. $(\mathrm{kS} / \mathrm{m})$ \\
\hline $98 \%$ metallic $\mathrm{NI}\left(\mathrm{R}^{2}: 0.97\right)$ & $38.20+/-0.10$ & $0.71+/-0.02$ & $-0.84+/-0.00$ & 83.5 \\
\hline Unaligned SWCNT ${ }^{22}$ & 33 & 3.2 & 0 & 69 \\
\hline $98 \%$ semi-conducting $N I\left(R^{2}: 0.99\right)$ & $159.2+/-65.7$ & $0.18+/-.05$ & $-0.98+/-.01$ & 26.8 \\
\hline 93\% Metallic SWCNT ${ }^{28}$ & 60.16 & .054 & -0.87 & 15.4 \\
\hline $94 \%$ semi-conducting SWCNT ${ }^{28}$ & 64 & 0.04 & -0.69 & 1.8 \\
\hline
\end{tabular}

well the microwave transport at room temperature. The previous transport measurements on sorted films ${ }^{11}$ concluded that the small fraction of metallic CNTs that remains in the sample after the sorting process dominates the electrical transport as current will bypass the highly resistive semi-conducting regions. However, this scenario seems to be unlikely due to the relative high DC conductivity of the $98 \%$ semi-conducting NI film. With room temperate conditions and atmospheric exposure, it is reasonable to expect that the electrons associated with semi-conducting SWCNTs are thermally activated into the conduction band and should

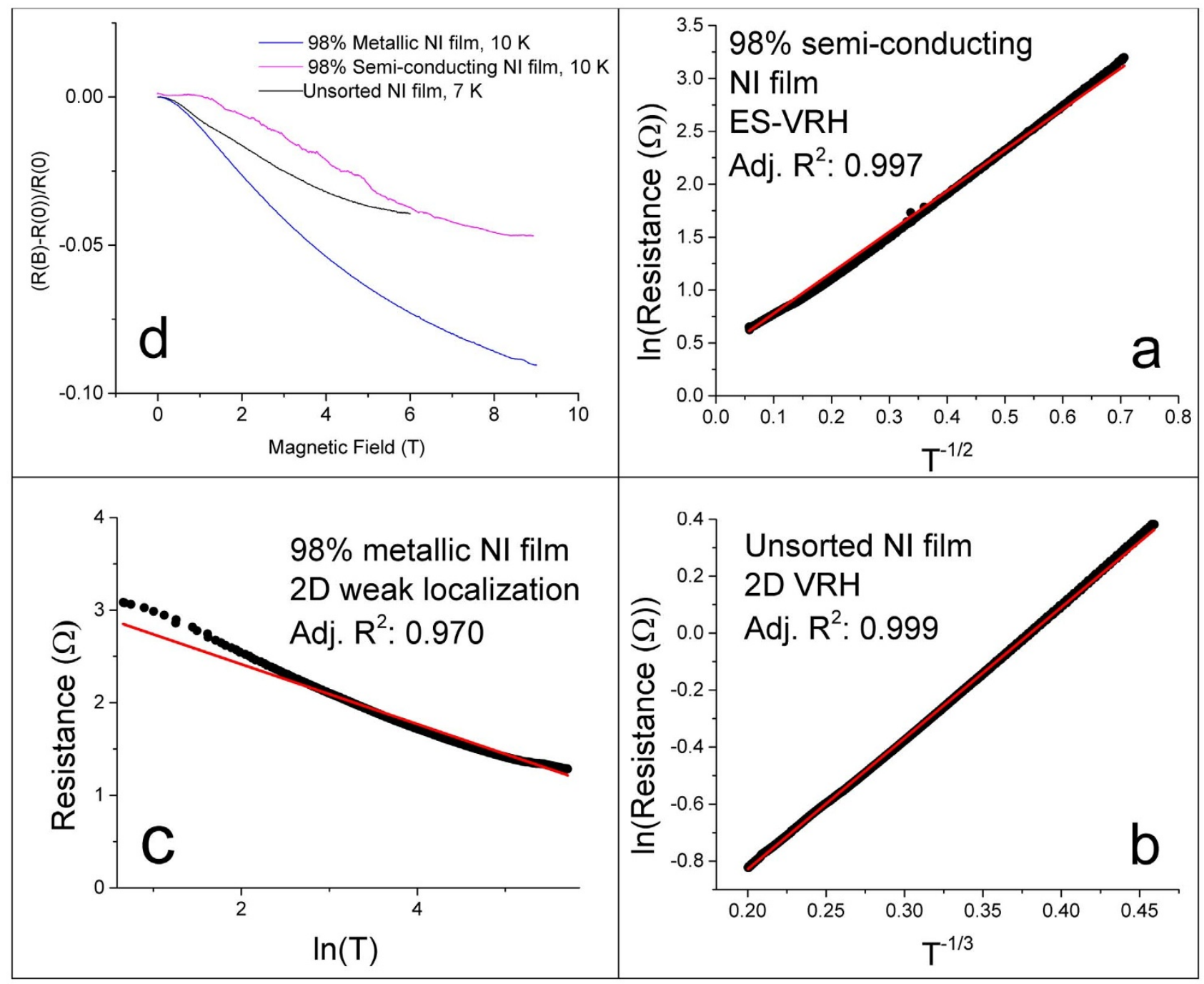

Figure 5 $\mid$ For the semi-conducting (a) and unsorted (b) NI films, they fit best to the ES-VRH and 2D VRH models respectively as shown with the natural log of resistance versus a dimensionality dependent temperature expression. The metallic sample (c) did not fit to any VRH model, but was the only one that to have a near linear resistance dependence with $\ln (\mathrm{T})$. This suggests $2 \mathrm{D}$ weak localization regulates the conductivity as found with metallic thin films. (d) Magneto-resistance (MR) measurements were performed to acquire localization length, but this was not possible because of a lack of positive MR in all three film concentrations. The plot is normalized resistance versus magnetic field. 
contribute to the transport. For the unsorted NI film, the 2D VRH can be explained by hopping between adjacent SWCNTs or bundles being the limiting mechanism of the overall conductivity ${ }^{43,44}$. This picture agrees with the mean free path equaling the SWCNT length as the microwave measurements suggest. The same applies with the $98 \%$ metallic NI film where even in weak localization the primary source of elastic scattering should be from the ends of the SWCNT.

We conducted Magneto-resistance (MR) measurements to $9 \mathrm{~T}$ (Fig. 5d), where there was the initial, well reported resistance drop due to of suppression of weak localization ${ }^{43-46}$. Surprisingly, for all three materials to include the $98 \%$ semi-conducting NI film, this was not accompanied by the familiar upturn in MR at higher field. This positive MR component is attributed to hopping between SWCNTs (or SWCNT bundles) and determines relevant VRH parameters such as localization length. Because no MR upturn was observed, we were not able to calculate a localization length or hopping distance. In past studies, only the most conductive, heavily doped, or most metallic SWCNT films did not have this positive MR upturn ${ }^{11,13,44}$. Despite the differences in metallic concentration, the room temperature, DC conductivity of each of the NI films were within an order of magnitude of each other due to the doping influence of the atmosphere. Presumably, atmospheric doping affects the charge transfer between SWCNT structures and the NI films appear to respond similarly with the magnitude of field reached. Possibly with higher field, the MR upturns would become apparent as suggested by a leveling in the MR for the $98 \%$ semi-conducting and unsorted NI films. While MR did not yield any quantitative parameters, it shows the influence of atmospheric doping with the SWCNTs.

Cryogenic measurements showed that the $98 \%$ semiconducting had the most disordered transport where conduction was limited to 1D hopping down the SWCNT. Next was the unsorted NI film limited by hopping from one SWCNT structure to the next. The metallic NI film had the least disorder and showed quantum transport limited by weak localization. Conductivity measurements up to $120 \mathrm{GHz}$ agree with the cryogenic results when using both the universal disorder and generalized Drude conduction models. The Drude conduction model, up until now only applied to the $\mathrm{THz}$ and optical wavelengths, fit the best to all three film concentrations and yielded realistic values for the transport parameters. Potentially, microwave measurements may be useful for rapid quality control in CNT production. A contactless microwave probe could calculate average CNT length, charge carrier density, and the degree of localization. DC conductivity could be continuously extrapolated without touching the CNT material. Internal fibre alignment as well may be calculated by measuring the anisotropy with changing the microwave polarization. These techniques combined with other traditional measurements such as Raman spectroscopy would be powerful combination for a rapid, contact-less quality control, a much needed tool for CNT programs attempting high quality, high quantity output.

Placing SWCNT films into practical micro-strip waveguides immediately shows the advantage in microwave transmission of sorted over unsorted without taking into account variable parameters such as film thickness or de-embedding techniques. This advantage was shown not just with unaligned NI films, but also with sorted UCAM fibres of predominantly metallic chirality that are internally longer length and aligned. While there is significant work on improving SWCNT assembly conductivity for high strength, DC applications, the advantages of SWCNT based conductors will be first utilized at high frequency situations where conductivity improves and the performance of traditional metals worsens-- particular in applications where weight is important such as EMI shielding or absorbers for aerospace applications $s^{47,48}$. For such high frequency scenarios, we now know sorted SWCNTs are much preferable to unsorted.

\section{Methods}

Material. NI films were purchased from NanoIntegris. From company provided thermogravometric analysis (TGA), all the NI films had a catalytic and amorphous carbon/residual surfactant impurity of $\sim 1 \%$ and $\sim 5 \%$ respectively. Raman spectroscopy done with a Renshaw Raman spectrometer yielded a radial breathing mode $(\mathrm{RBM})$ peak and a G:D ratio of $\sim 23$. From company provided Atomic Force Microscopy (AFM), the NI films have a length spanning from $100 \mathrm{~nm}$ to $4 \mu \mathrm{m}$, with an average of $0.5 \mu \mathrm{m}$ of the $98 \%$ metallic and $1 \mu \mathrm{m}$ for the $98 \%$ semi-conducting and unsorted. Compared to the NI films, the UCAM material has higher catalytic and amorphous carbon impurities -- $20 \%$ and $12 \%$ respectively. The UCAM materials, however, have the attribute of internal CNT alignment (as verified by SEM) and length ${ }^{2}$-- estimated at $\sim 1 \mathrm{~mm}$, which is about 1000 times longer than the SWCNTs in the NI films. The UCAM material has RBMs in its Raman spectra and a G:D ratio of $\sim 20$. Details on the predominantly metallic UCAM fibre is previously written ${ }^{6}$.

Micro-strip. Micro-strip transmission line of $50 \Omega$ characteristic impendence was manufactured by SMS Microwave Ltd. The micro-strip was completely shielded with a fitted grounding plane to mitigate radiation losses. Ports were Anritsu Type K connectors. A section of the micro-strip's central conductor was cut and removed and a SWCNT film with equal dimensions was placed in its stead. Gingerly applied colloidal silver paint connected the film to the micro-strip. S-parameters were collected with a SOLT calibrated Anritsu VectorStar Vector Network Analyzer (VNA). We limit consideration up to $10 \mathrm{GHz}$ with a shortest wavelength at $\sim 23 \mathrm{~mm}$. This ensures the CNT film itself, with length not greater than $3 \mathrm{~mm}$, is small enough to be treated as a lumped parameter resistor in the micro-strip transmission line. Experimental testing met ISO/IEC 17025 requirements with the maximum error in the insertion loss measurement being better than $2.6 \%$ across the frequency spectrum.

Expected performance of the micro-strip with a typical lumped parameter resistor was predicted using the measured DC resistance and a simple micro-strip transmission line model from Microwave Office, a commercial microwave circuit simulator (see insets of Fig. 2). A frequency dependent resistance was calculated by tuning the lumped parameter resistor in the transmission line model to match the measured insertion loss at a particular given frequency. This approach yields absolute resistance values, which were turned into a sheet conductance.

The DC multimeter measurement for the micro-strip was two probe for fair comparison because the VNA's S-parameter measurement does not eliminate contact resistance between the CNTs and colloidal silver paint. The possibility that the conductivity increase came from simply capacitivly bypassing the silver paint contacts was ruled out. Four probe measurement shows contact resistance from the metallic and unsorted materials was low at $\sim 2 \%$ and longer length samples for each film (with a smaller percentage contact resistance) had similar microwave behavior. Note that while the sorted NI films had a higher conductivity then the unsorted, the film thickness of the unsorted was greater. This led to the films having similar DC resistance values. For the $98 \%$ metallic, $98 \%$ semi-conducting, and unsorted NI films, $\sim 1 \mathrm{~mm}$ long samples: $6.3,7.7$, and $5.2 \Omega$.

Waveguide. For broadband, continuous conductivity measurement up to $120 \mathrm{GHz}$, we used a standard microwave transmission/reflection technique ${ }^{49,50}$ that sandwiches material (various SWCNT films) between two rectangular waveguides such that the film is incident to the wave propagation. Here, the entire electromagnetic wave traveling down one end of the waveguide to the other must interact with the material under test, where a fraction of the wave energy is reflected back to the source and another fraction passes through to the end. This is in contrast to the more convoluted micro-strip situation above where the material under test is only at the edge boundary of the electromagnetic wave. All four, complex S-parameters were measured by a 2 port Anritsu VectorStar VNA after a TRL calibration (performed in waveguide). This VNA is fitted with millimeter wave modules that attach directly to the waveguides and enable broadband coverage up to $120 \mathrm{GHz}$. The waveguides were either K or W band. The NI films under consideration are rigid and self-supporting on the physical dimensions considered here and were laid flat across the waveguide's pathway. Because the material is a collection of bundles of CNTs, it can be compressed to a degree around its edges where the two waveguides attach together. For consistency, the two waveguides were pressed and bolted together (with the film sandwiched between them) and then separated (with the film immobilized and still attached to one of the waveguides) multiple times. This was repeated until it was certain the Sparameters were stable from this pressing/separation procedure. The S-parameters required de-embedding procedures to remove the impact of leakage around the edges of the sample. The admittances associated with this gap can be calculated ${ }^{51}$ and deembedded using a commercial circuit simulator. The values were validated with measurements of metal shims with geometries similar to those of the CNT samples.

The de-embedded S-parameters were directly converted into a frequency dependent permittivity and permeability using the Nicolson, Ross, Weir (NRW) equations ${ }^{49,50}$. The NRW equations, obtained by considering the continuity of the electric field at the interfaces of the material under test and the wave guides, explicitly relate permittivity/permeability with the VNA's S-parameters. This technique however has phase ambiguity in the transmission coefficient and must be supplemented with a phase wrapping procedure or by equating the phases of two transmission coefficients calculated from S-parameters measured sufficiently close in frequency ${ }^{49}$ The microwave conductivity is related to the complex permittivity by the well known relationship for a lossy conductor $\varepsilon=\varepsilon^{\prime}-i \sigma(\omega) / \omega$ where $\varepsilon$ is the complex permittivity and $\varepsilon^{\prime}$ is the real part. The NRW algorithm requires the film's thickness, which was obtained by averaging cross-sections under a SEM. There are edge-of-band 
distortions that contribute to the 'smile' appearance at the ends of the trace; that is, cutoff effects at the low frequency edges and higher order mode propagation at the upper frequency edge. The shaded regions in Fig. 4 are the estimated spread in conductivity brought on by the considerable variance in film thickness, the prevailing source of error in the measurement.

Cryogenic transport. In a four probe arrangement, samples were silver painted onto an insulating support at the contact points with approximately $1 \mathrm{~mm}$ between inner leads. Anchoring the sample to the support with adhesive increased setup robustness, but was found to modify the transport behavior and was not used. The samples were placed into a Quantum Design Physical Properties Measurement System (PPMS). Resistance versus temperature data was fitted to Mott's variable range hopping with $R(T) / R_{0}=e^{\left(T_{M} / T\right)^{1 /(d+1)}}$ with $T_{M}$ being the characteristic Mott temperature and $d$ being the discrete dimensionality of the electron hopping.

1. Iijima, S. Helical microtubules of graphitic carbon. Nature 354, 56-58 (1991).

2. Koziol, K. et al. High performance carbon nanotube fiber. Science 318, 1892-1895 (2007).

3. Windle, A. \& Koziol, K. Continuous spinning of carbon nanotube fibres: structure control and properties. Sen'i Gakkaishi Society of Fiber Science and Technology of Japan 63, 361-364 (2007).

4. Paukner, C. \& Koziol, K. Ultra-pure single wall carbon nanotube fibers continuously spun without promoter. Sci. Rep. 4, 3903; doi:10.1038/srep03903 (2014).

5. Paukner, C. Structural Control of Carbon Nanotubes in the Continuous Fibre Spinning Process. MRS fall 2012, Boston, MA.

6. Sundaram, R., Koziol, K. \& Windle, A. Continuous direct spinning of fibers of single-walled carbon nanotubes with metallic chirality. Adv Mater 23, 5064-5068 (2011).

7. Behabtu, N. et al. Strong, Light, Multifunctional Fibers of Carbon Nanotubes with Ultrahigh Conductivity. Science 339, 182 (2013).

8. Fischer, J. et al. Metallic resistivity in crystalline ropes of single-wall carbon nanotubes. Phys. Rev. B. 55, 4921 (1997).

9. Kaiser, A., Dusberg, G. \& Roth, S. Heterogeneous model for conduction in carbon nanotubes. Phys. Rev. B 57, (1998).

10. Nirmalra, P., Lyons, P., De, S., Coleman, J. \& Boland, J. Electrical Connectivity in Single-Walled Carbon Nanotube Networks. Nano Lett. 9, 3890 (2009).

11. Yanagi, K. et al. Transport Mechanisms in Metallic and Semi-conducting SingleWall Carbon Nanotube Networks. ASC Nano 4, 4027-4032 (2010).

12. Xu, H. et al. Microwave shielding of transparent and conducting single-walled carbon nanotube films. Appl. Phys. Lett. 90, 183119 (2007).

13. Salvato, M. et al. Effect of potassium doping on electrical properties of carbon nanotube fibers. Phys. Rev. B. 84, 233406 (2011).

14. Hersam, M. Nanotubes keep rolling on. Nature Nanotechnology 4, 465 (2009).

15. Hároz, E. et al. Fundamental optical processes in armchair carbon nanotubes. Nanoscale 5, 1411-1439 (2013).

16. Arnold, M., Green, A., Hulvat, J., Stupp, S. \& Hersam, M. Sorting carbon nanotubes by electronic structure using density differentiation. Nature Nanotech. 1, 60-65 (2006).

17. Highstrete, C., Lee, M., Talin, A. \& Vance, A. Microwave conductance spectra of single-walled carbon nanotube arrays. Appl. Phys. Lett. 95, 203111 (2009).

18. Xu, H., Zhang, S., Anlage, S., Hu, L. \& Grüner, G. Frequency- and electric-fielddependent conductivity of single-walled carbon nanotube networks of varying density. Phys. Rev. B 77, 075418 (2008).

19. Dyre, J. The random free energy barrier model for ac conduction in disordered solids. J. Appl. Phys. 64, 2456 (1988).

20. Dyre, J. Universality of ac conduction in disordered solids. Rev. Mod. Phys. $\mathbf{7 2}$ 873-892 (2000).

21. Kilbride, B. et al. Experimental observation of scaling laws for alternating current and direct current conductivity in polymer-carbon nanotube composite thin films. J. Appl. Phys. 92, 4024 (2002).

22. Hilt, O., Brom, H. \& Ahlskog, M. Localized and delocalized charge transport in single-wall carbon nanotube mats. Phys. Rev. B 61, R5129-R5132 (2000).

23. Jeon, T. et al. Optical and electrical properties of preferentially anisotropic singlewalled carbon-nanotube films in terahertz region. J. Appl. Phys. 95, 5736 (2004).

24. Jeon, T. et al. Terahertz conductivity of anisotropic single walled carbon nanotube films. Appl. Phys. Lett. 80, 3403 (2002).

25. Seo, M. et al. Terahertz electromagnetic interference shielding using single-walled carbon nanotube flexible films. Appl. Phys. Lett. 93, 231905 (2008).

26. Jeon, T. et al. Terahertz absorption and dispersion of fluorine-doped single-walled carbon nanotube. J. Appl. Phys. 98, 034316 (2005).

27. Kampfrath, T. et al. Optical response of single-wall carbon nanotube sheets in the far-infrared spectral range from $1 \mathrm{THz}$ to $40 \mathrm{THz}$. Phys. Stat. sol. (b) $\mathbf{2 4 4}$, 3950-3954 (2007).

28. Beard, M., Blackburn, J. \& Heben, M. Photogenerated free carrier dynamics in metal and semiconductor single walled carbon nanotube films. Nano Lett. 8 , 4238-4242 (2008).

29. Ugawa, A., Rinzler, A. \& Tanner, D. Far-infrared gaps in single-wall carbon nanotubes. Phys. Rev. B 60, R11305-R11308 (1999).

30. Pekker, A. \& Kamaras, K. Wide-range optical studies on various single-walled carbon nanotubes: Origin of the low-energy gap. Phys. Rev. B 84, 075475 (2011).
31. Akima, N. et al. Strong Anisotropy in the Far-Infrared Absorption Spectra of Stretch-Aligned Single-Walled Carbon Nanotubes. Adv. Mater. 18, 1166-1169 (2006).

32. Shuba, M. Experimental evidence of localized plasmon resonance in composite materials containing single-wall carbon nanotubes. Phys. Rev. B 85, 165435 (2012).

33. Zhang, Q. et al. Plasmonic Nature of the Terahertz Conductivity Peak in SingleWall Carbon Nanotubes. Nano Lett. 13, 5991-5996 (2013).

34. Alshehri, A. et al. Electrical performance of carbon nanotube-polymer composites at frequencies up to $220 \mathrm{GHz}$. Appl. Phys. Lett. 99, 153109 (2001).

35. Ruben Esteban, R., Borisov, A., Nordlander, P. \& Aizpurua, J. Bridging quantum and classical plasmonics with a quantum-corrected model. Nat. Commun. 3, 825 (2012).

36. Prodan, E., Radloff, C., Halas, N. \& Nordlander, P. A Hybridization model for the plasmon response of complex nanostructures. SCIENCE 302, 409 (2003).

37. Luk'yanchuk, B. et al. The Fano resonance in plasmonic nanostructures and metamaterials. Nature Mater. 9, 707-715 (2010).

38. Smith, N. Classical generalization of the Drude formula for the optical conductivity. Phys. Rev. B 64, 155106 (2001).

39. Turner, G., Beard, M. \& Schmuttenmaer, C. Carrier Localization and Cooling in Dye-Sensitized Nanocrystalline Titanium Dioxide. J. Phys. Chem. B 106, 11716-11719 (2002).

40. Collins, P., Bradley, K., Ishigami, M. \& Zettl, A. Extreme Oxygen Sensitivity of Electronic Properties of Carbon Nanotubes. Science 287, 1801-1804 (2000).

41. Bradley, K. et al. Is the Intrinsic Thermoelectric Power of Carbon Nanotubes Positive? Phys. Rev. Lett. 85, 4361-4364 (2000).

42. Sumanasekera, G., Adu, C., Fang, S. \& Eklund, P. Effects of Gas Adsorption and Collisions on Electrical Transport in Single-Walled Carbon Nanotubes. Phys. Rev. Lett. 85, 1096 (2000).

43. Takano, T., Takenobu, T. \& Iwasa, Y. Enhancement of Carrier Hopping by Doping in Single Walled Carbon Nanotube Films. J. Phys. Soc. Jpn. 77, 124709 (2008).

44. Vavro, J., Kikkawa, J. \& Fischer, J. Metal-insulator transition in doped single-wall carbon nanotubes. Phys. Rev. B. 71, 155410 (2005).

45. Yosida, Y. \& Oguro, I. Variable range hopping conduction in bulk samples composed of single-walled carbon nanotubes. J. Appl. Phys. 86, 999 (1999).

46. Kim, G. et al. Magnetoresistance of an entangled single-wall carbon nanotube network. Phys. Rev. B 58, 16064-16069 (1998).

47. Micheli, D., Apollo, C., Pastore, R. \& Marchetti, M. X-Band microwave characterization of carbon-based nanocomposite material, absorption capability comparison and RAS design simulation. Compos. Sci. Technol 70, 400-409 (2010).

48. Micheli, D. et al. Broadband electromagnetic absorbers using carbon nanostructure-based composites. IEEE T MICROW THEORY 59, 2633-2646 (2010).

49. Chen, L. Microwave Electronics Measurement and Materials Characterization (Wiley, 2004).

50. Barker, J., Vanzura, E. \& Kissick, W. Improved technique for determining complex permittivity with the transmission/reflection method. IEEE T. Microw. Theory 38, 1096 (1990).

51. N. Marcuvitz, E. Waveguide Handbook (Dover, 1965).

\section{Acknowledgments}

We thank Martin Sparks and Krste Pangovski at the Institute for Manufacturing, Cambridge for assistance with laser cutting and optical measurement. We thank Simon Griggs at the Department of Material Science, Cambridge for assistance with SEM cross sections. We also thank Paul Holes, John Dunstan, and Bob Buxton of Anritsu Corporation for VNA support. Thanks to Steve Jenkins and John Howard from SMS limited on micro-strip design and fabrication.

\section{Author contributions}

J.M. performed contactless microwave measurements, de-embedding procedures, and conductivity extraction. L.K. performed micro-strip measurements and, with T.G. analysis. M.E. performed cryogenic measurements and analysis. J.B. wrote the paper, participated in all the various experiment components, and project planning. N.Y., M.B. and K.K. provided project oversight and direction. All the authors discussed the results and reviewed the manuscript.

\section{Additional information}

Competing financial interests: The authors declare no competing financial interests.

How to cite this article: Bulmer, J.S. et al. Microwave Conductivity of Sorted CNT Assemblies. Sci. Rep. 4, 3762; DOI:10.1038/srep03762 (2014).

This work is licensed under a Creative Commons Attribution-

NonCommercial-ShareAlike 3.0 Unported license. To view a copy of this license, visit http://creativecommons.org/licenses/by-nc-sa/3.0 